\title{
Polymer-based technology platform for robust electrochemical sensing using gold microelectrodes
}

\author{
M. Kuphala, ", C. A. Mills ${ }^{b}$, H. Korri-Youssoufic ${ }^{c}$, J. Samitier ${ }^{a, d, e}$
}

${ }^{a}$ Institute for Bioengineering of Catalonia (IBEC), Barcelona Science Park, C/Baldiri Reixac 10-12, 08028 Barcelona, Spain

${ }^{\mathrm{b}}$ Advanced Technology Institute, University of Surrey, Guildford, GU2 8HD, United Kingdom

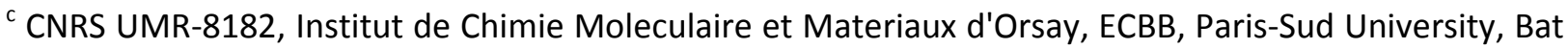
420, 91405 Orsay, France

${ }^{d}$ Centro de Investigacion Biomedica en Red, Bioingenieria, Biomateriales y Nanomedicina (Ciber-BBN), 50018 Zaragoza, Spain

e Department of Electronics, University of Barcelona, C/Mart i Franque's 1, 08028 Barcelona, Spain

\begin{abstract}
Rapid and inexpensive development of electrochemical sensors with good exploitation potential may be produced using a polymer as a substrate material. However, fabrication of polymer-based sensors is challenging. Using photolithography and etching of gold-coated poly(ethylene-2,6naphthalate) (PEN), we have succeeded in fabricating disk-shaped and interdigitated microelectrodes $(\mu \mathrm{Es})$. The electrodes have an excellent adhesion to the polymer and are encapsulated using a novel room-temperature process, applicable for low-cost, high-throughput fabrication. The PEN surface has been characterized in respect of wettability, surface energy and surface roughness. Finally, the electrodes give stable and reproducible electrochemical impedance spectroscopy and cyclic voltammetry responses, using the redox couple ferrocyanide and ruthenium hexamine. The results demonstrate the robustness and functionality of the polymer-based sensor platform with minimum feature sizes of $6 \mu \mathrm{m}$.
\end{abstract}

Keywords: Poly(ethylene naphthalate), Photolithography, Microelectrodes, Interdigitated electrodes, Electrochemical characterization, Electrochemical sensor

"Corresponding author.Tel.: +34 686557949

Email address: mathias@kuphal.eu (M. Kuphal) 


\section{Introduction}

Biosensor development is arguably considered to be one of the main driving forces behind the diagnostic market and will reduce the burden of healthcare and environmental resources dramatically [1].

To ensure success, new materials with maximum functionality, coupled with suitable properties and inexpensive fabrication costs are needed. This work is a contribution to the field of product orientated development of biosensors. It describes a technology for robust electrochemical sensing using a polymer as a substrate material. This allows a rapid and low cost transition into clinical validation testing for further commercialization of a device.

Fabricating robust polymer-based devices is challenging, due to the compromise between processing temperature and device performance [2]. In particular, two considerations stand out. On the one hand, choosing to use a polymer as a substrate material is usually accompanied by the requirement to develop a fabrication process. On the other hand, silicon-based technologies are considered one of the most advanced fabrication processes. However, polymers are a competitive alternative to inorganic materials such as silicon [3].

Some important advantageous characteristics of polymers are a low price, light weight, flexibility, high impact resistance and ease of surface manipulation (mechanically and chemically). The challenges to surmount when using polymer substrates relate to the metal-to-polymer adhesion, thermal stability, solvent resistance and thermal expansion [4]. In particular, a good metal-to-polymer adhesion and good resistance to chemical species are fundamental for the reproducible performance of an electrochemical sensor.

The work presented here, details a technology for producing robust electrochemical biosensors. The biosensors use a thin gold ( $\mathrm{Au}$ ) layer deposited on poly(ethylene-2,6-naphthalate) (PEN) which is sequentially patterned to produce electrodes and passivated to define active sensing regions. The Au microelectrodes $(\mu \mathrm{E})$ are characterized by electrochemical spectroscopy (EIS), cyclic voltammetry (CV) and chronoamperometry $(\mathrm{CA})$, which are commonly used electrochemical biosensing techniques $[5,6]$. Electrochemical devices can be characterized in a number of ways [7-10], further optimization techniques can be found in the literature [11-13]. PEN is a polyester and belongs to the group of thermoplastic semi-crystalline polymers [14]. It has a good resistance to UV light, alkalis and acids and is certified for long term electrical use at $155^{\circ} \mathrm{C}$, but starts to shrink significantly at $190^{\circ} \mathrm{C}$. These properties make PEN compatible for microfabrication. PEN was chosen instead of other polymers such as poly(ethylene terephthalate) (PET) and poly(imide) (PI), because it outperforms PET and it is cheaper 
than PI (approx. $16 \%$, Goodfellow) with better properties like good clarity, less permeability to water and oxygen, less thermal expansion and better moisture absorption [15-18]. The substrate material, PEN, is a leading candidate for organic thin film transistors and organic light emitting diodes [2]. In the literature there are a number of reported fabrication processes available to pattern materials on PEN, including direct/lift-off photolithography [4], roll-to-roll photolithography [19], digital (mask) lithography [20], hot embossing lithography [21] and stencil lithography [22]. Materials used for patterning include gold [22], indium tin oxide [23], silicon thin films [24] and silver [21]. PEN-based biosensor devices, reported elsewhere $[25,26]$, have a minimum feature size of $20 \mu \mathrm{m}$, or the fabrication method is not described in detail. Fully described here is the production of $\mu$ Es of $6 \mu \mathrm{m}$ width with a high chemical and electrochemical robustness.

\section{Materials and equipment}

Aqueous solutions were prepared using Milli-Q reagent water (DI water, Millipore Corp.). DI water and formamide (99.5\%, Sigma-Aldrich) were used for contact angle measurements on the substrate material PEN (125 $\mu \mathrm{m}$ thick, ref. ES36 1090, Goodfellow) to determine its wettability and surface energy, which was estimated applying Owens-Wendt theory [27]. Ethanol (70\%, Sigma-Aldrich), isopropanol (99.5\%, Sigma-Aldrich), acetone (99.9\%, Sigma-Aldrich) and Piranha solution $\left(\mathrm{H}_{2} \mathrm{SO}_{4} / \mathrm{H}_{2} \mathrm{O}_{2}\right)$ were used to test the electrode fabrication stability. AZ726 MIF developer (MicroChemicals $\mathrm{GmbH}$ ), gold etch solution potassium iodine (type TFA, Transene Company Inc.) and for resist stripping N-methyl-2pyrrolidone (NMP, MicroChemicals $\mathrm{GmbH}$ ) were used for microfabrication. Sulfuric acid $\left(\mathrm{H}_{2} \mathrm{SO}_{4}, 95\right.$ \%, Sigma-Aldrich) dissolved in DI water (0.1 M) were used for electrochemical cleaning of the electrodes. Potassium ferrocyanide ( $\mathrm{K} 4 \mathrm{Fe}(\mathrm{CN}) 6,99.5 \%$, Sigma-Aldrich) dissolved in phosphate buffered saline (PBS, Sigma- Aldrich) (5 mM) and ruthenium hexaminetrichloride (RuHex, 262005-1G, Sigma-Aldrich) dissolved in $1 \mathrm{M}$ potassium chloride $(\mathrm{KCl}$, Sigma-Aldrich) $(10 \mathrm{mM})$ solutions were used for electrochemical test measurements. Female crimp connectors (Ref. 481-487, RS), Araldite epoxy glue (Ref. 106486, Farnell) and metal bars (Ref. 547-3188, RS) were used for device connection. For comparison of the PEN surface characterization results, Poly(imide) (Kapton HN, ref. 667-985-89, Goodfellow) was used. The 60-7155 UV epoxy (Epoxies Etc., Cranston, RI 02921 USA) was used for passivation. Photolithography was completed using a spin coater (WS-650MZ-23NPP/LITE, Laurell Technologies Corporation) and a mask aligner (MJB4, SUSS MicroTec), while the polymer was fixed for spin coating on a borosilicon float glass support ( $1 \mathrm{~mm}$ thick, diameter of $100 \mathrm{~mm}$, Siegert Consulting e.K., Germany). A Bio- Logic potentiostat (EPP-4000) was used for CV and CA measurements and an Autolab potentiostat (PGstat 20) for EIS measurements. The surface roughness of PEN was measured 
using an atomic force microscope (AFM, Veeco) and the Au metal layer thicknesses were determined using a mechanical profilometer (Dektak $6 \mathrm{M}$, tip radius of $5 \mu \mathrm{m}$, measurement resolution of $0.167 \mu \mathrm{m}$, Veeco) and AFM. The different active areas have been determined by pixel measurements based on optical images using phase-contrast microscopy.

\section{Methods}

\subsection{Au patterning: photolithography with wet etching}

An iso-directional Au wet etch compensation of $3.5 \mu \mathrm{m}$ is taken into account in the photomask design; consequently structure sizes are increased and gap sizes are decreased by $3.5 \mu \mathrm{m}$ in the photomask.

Metal deposition. The PEN was cleaned with isopropanol and DI water and then fixed to the sputter apparatus support plate by poly(imide) adhesive tape. Planar contact to the support is important to ensure homogeneous metal deposition by physical vapor deposition (PVD, sputtering). Experiments showed that an extra metal layer (e.g. chrome or titanium), to promote metal adhesion, is not necessary, as the gold adheres directly to the polymer. Argon is used as a plasma gas with a vacuum pressure lower than $1 \times 10^{-6}$ bar. The spinning support of the sputterer used, is a custom made plate with diameter of $24 \mathrm{~cm}$, which allows the use of substrate sizes of $11 \mathrm{~cm}$ by $21 \mathrm{~cm}\left(231 \mathrm{~cm}^{2}\right)$. The time for deposition of a $250 \mathrm{~nm}$ thin Au layer is $15 \mathrm{~min}$.

Photolithography with wet etching. A typical photolithography process is applied using AZ5214 E photoresist, which is spin-coated onto the Au layer for $20 \mathrm{~s}$ at $2000 \mathrm{rpm}$. The applied photoresist is initially soft baked on a hotplate for $120 \mathrm{~s}$ at $95^{\circ} \mathrm{C}$. The photoresist layer is then exposed to UV light with energy of $200.16 \mathrm{~mJ} / \mathrm{cm}^{2}$ for $7.2 \mathrm{~s}$ through a positive mask with a light intensity of $27.8 \mathrm{~mW} / \mathrm{cm}^{2}$. Exposure is completed in the near-UV 400 range (wavelength $=365 \mathrm{~nm}, 350 \mathrm{~W}$ mercury lamp), with the mask and the photoresist layer in soft contact. Resist development is achieved by immersion in developer for $30 \mathrm{~s}$. The etching is completed by immersion in TFA for $70 \mathrm{~s}$. The remaining photoresist is removed by immersion in NMP for $40 \mathrm{~s}$. Then, the substrate is rinsed in DI water for $30 \mathrm{~s}$ and dried by nitrogen gas.

\subsection{Passivation layer patterning: photolithography}

To open the active areas of the Au electrodes, and to pacify the redundant electrode areas, 60-7155 UV epoxy is applied via a photolithography process. The photosensitive epoxy is spin-coated onto the 
PEN substrate for $20 \mathrm{~s}$ at $2000 \mathrm{rpm}(15.7 \pm 2.9 \mu \mathrm{m}$ thick). Then, the epoxy is cured by exposing to UV light for $160 \mathrm{~s}$ through a positive mask in non-contact mode. The mask aligner is used in constant intensity mode using a light intensity of $33 \mathrm{~mW} / \mathrm{cm}^{2}$. The light energy is $5.28 \mathrm{~W} / \mathrm{cm}^{2}$.

The exposed epoxy areas are then removed during resist development by rinsing in ethanol for $10 \mathrm{~s}$. After development, the substrate is rinsed in DI water for $20 \mathrm{~s}$ and dried under nitrogen gas. The described procedure works for thinner epoxy layers down to $3.6 \pm 0.6 \mu \mathrm{m}$, spin coated for $8000 \mathrm{rpm}$ (figure 2).

\subsection{Device connection}

Devices are connected using encapsulated female Au crimp connectors. Before encapsulation with an epoxy, silver coated bars are soldered to the crimp connection. Wires can be connected to these inline connectors. The glue is painted on the crimp connectors and cured for $1 \mathrm{~h}$ at $100^{\circ} \mathrm{C}$ in an oven. The encapsulation is performed to avoid damage of the gold pads by crimp connector movement. This ridged connection also improves the device performance, because the temperature curing dehydrates the Au-polymer material, which increases the adhesion strength of metallic films to polymer substrates [28].

\subsection{Electrochemical characterization}

The devices developed to characterize the electrochemical behavior of the fabricated Au $\mu E s$ are $6 \mu \mathrm{m}$ interdigitated electrodes (IDE) and $30 \mu \mathrm{m}$ disk electrodes, used to perform EIS and CV/CA, respectively. These electrode con-figurations are the most studied and commonly used for these sensing techniques $[29,30]$. The width of the IDE elements is $6 \mu \mathrm{m}$ and each IDE has 53 electrodes pairs (IDE 0653, figure 3.c). The disk $\mu \mathrm{E}$ devices consist of two working electrodes (WE), one counterelectrode (CE), and one pseudo reference electrode (RE). This general design is adapted from [31] (figure 3.a). The active areas are ideally $707 \mu \mathrm{m}^{2}$ (WE, figure 3.b), $0.035 \mathrm{~mm}^{2}$ (RE) and $0.57 \mathrm{~mm}^{2}$ (CE). Due to alignment errors and feature size variations, the WE active area can change between different devices up to a factor of three and the biggest measured active WE area is $2302 \mu \mathrm{m}^{2}$.

Electrochemical cleaning procedure. Prior to characterization, each gold electrode is individually cleaned electro- chemically with $0.1 \mathrm{M} \mathrm{H}_{2} \mathrm{SO}_{4}$, cycled between $0 \mathrm{~V}$ and $1.6 \mathrm{~V}$ at a scan rate of $1 \mathrm{Vs}^{-1}$ for 15 to maximum 80 cycles. 
EIS measurements. The impedance behavior of the gold IDES is characterized in $5 \mathrm{mM} \mathrm{K} 4 \mathrm{Fe}(\mathrm{CN})_{6}$ $\left(\mathrm{FE}^{2+} / \mathrm{FE}^{3+}\right.$ redox couple) using a silver-silver chloride $(\mathrm{Ag} / \mathrm{AgCl})$ as reference electrode. The IDES are connected as WE and CE to the potentiostat. The results are analyzed using Nyquist plots with an associated equivalent circuit (EC). The EC parameters are fitted using Zview (3.22d, ECHEM). All impedance measurements are performed at open circuit potential and the applied potential is $10 \mathrm{mV}$ with a frequency range of $100 \mathrm{kHz}$ to $10 \mathrm{mHz}$. To ensure measurement stability, the experiments are repeated three times and the standard deviation is less than $5 \%$.

$\mathrm{CV}$ and CA measurements are carried testing every working electrode individually to determine the microelectrode behavior (diffusion limited processes). Two different experimental setups were applied. One is called "external setup" and the other one is named "internal setup". The external setup makes use of an outlying (external) $\mathrm{Ag} / \mathrm{AgCl}$ reference electrode in $3 \mathrm{M} \mathrm{KCl}$ and a platinum wire counter electrode are used. The internal setup uses an "on chip" gold quasi reference and counter electrode. For the study of the macro electrode behavior, the "on chip" gold CE is used as a working electrode. All measurements are performed in a faraday cage and the measurement results are expressed as the mean \pm standard deviation.

The $\mathrm{CV}$ is accomplished in $10 \mathrm{mM}$ RuRex. The CA current responses are measured in the range of $1 \mathrm{mM}$ to $20 \mathrm{mM}$ RuRex in $5 \mathrm{ml} \mathrm{KCl}(1 \mathrm{M})$. Consecutive aliquots of $100 \mathrm{mM}$ RuRex are added to the $\mathrm{KCl}$ solution until the final concentration of RuRex is achieved. After every addition, the solution is stirred for approximately $60 \mathrm{~s}$ to obtain the same initial conditions for each measurement.

\section{Characterization results and discussion}

The measured contact angle of DI water on PEN is $75.1^{\circ} \pm 1.6^{\circ}(n=9)$, which classifies PENs wettability as hydrophilic, a characteristic desired for micro-fabrication processes. PEN has a low value of partial polar energy $\left(8.0 \mathrm{~mJ} / \mathrm{m}^{2}\right)$, which indicates that only small polar interactions can be formed, which are required for an adhesive coating of a thin metal layer [32]. The total surface energy of PEN is $38.0 \mathrm{~mJ} / \mathrm{m}^{2}$. For comparison, $\mathrm{PI}$ has a slightly higher surface energy with $50.6 \mathrm{~mJ} / \mathrm{m}^{2}$ (polar surface energy of $7.7 \mathrm{~mJ} / \mathrm{m}^{2}$ ). 
A typical PEN surface is smooth $\left(R^{1}=8.5 \pm 4.2 \mathrm{~nm}\right)$, but may contain shallow scratches, which have a negligible influence on the adhesion stability. The surface smoothness is required to ensure adhesion of conductive coatings [15]. The surface roughness of $A u$ on PEN is $R Z=9.6 \pm 2.8 \mathrm{~nm}(n=6)$. This roughness increases the overall Au surface area about $1.03 \%$. The measured metal thickness discrepancy is approximately $20 \%$ over the first $5 \mathrm{~cm}$, decreasing concentrically from the center (due to the spinning support plate). The gold can be patterned with a minimum feature size of $6 \pm 1 \mu \mathrm{m}$. The total surface energy of the hydrophilic Au surface on PEN is $30.5 \mathrm{~mJ} / \mathrm{m}^{2}$ (DI water contact angle of $81.0^{\circ}$ $\left.\pm 2.2^{\circ},(n=3)\right)$. For comparison, the partial polar surface energy $\left(5.5 \mathrm{~mJ} / \mathrm{m}^{2}\right)$ of Au on PEN is higher than that for Au on $\mathrm{PI}\left(0.8 \mathrm{~mJ} / \mathrm{m}^{2}\right)$, which indicates that more polar interactions can be formed to other coatings.

The gold structures are not damaged by immersion in ethanol, isopropanol and acetone for more than $72 \mathrm{~h}$. Even a rinse for $30 \mathrm{~s}$ with Piranha solution (sulfuric acid + hydrogen peroxide) does not destroy the $6 \mu \mathrm{m}$ Au pattern. In addition, the gold electrodes resist pull-off tests with scotch adhesive tape and survive bending tests. Both tests results in no metal delamination or fractures. The passivation layer technology has the same chemical resistance and areas of $0.04 \mathrm{~mm}^{2}(200 \mu \mathrm{m} \times 200 \mu \mathrm{m})$ can be opened to expose the required active electrode area.

Standard passivation materials applied in industry are silicon nitride [33], silicon dioxide [34], parylene [35], poly-imide [36] and benzocyclobutene [37]. The deposition of these materials are performed by plasma enhanced chemical vapor deposition (PECVD) or spin coating at temperatures higher than $200^{\circ} \mathrm{C}$ [38], which excludes them for the use with PEN. Alternatively, functionalized SU-8 [39] could be applied. However, we have developed a room-temperature fabrication process, which has various advantages over SU-8 passivation. First, no extra functionalization is needed and layer thicknesses lower than those using SU-8 can be produced. Further, no additional baking steps (as for SU-8 at temperatures from 65 to $200{ }^{\circ} \mathrm{C}$ ) are required, which shortens the manufacturing time drastically and provides stress relief between the passivation layer and the substrate material. As can be seen, this passivation process can be applied for materials where a low process temperature is crucial.

Figure 4.a shows the EIS result using IDE with $\mathrm{K} 4 \mathrm{Fe}(\mathrm{CN}) 6$ as an analyte. The Nyquist plot shows a semicircle at high and medium-frequencies and a second depressed semicircle at low frequencies. The second low-frequency semicircle mainly reflects mass-transport limitations due to adsorption of

\footnotetext{
${ }^{1} \mathrm{RZ}$ is the average difference in height between the five highest peaks and five lowest valleys relative to the mean plane.
} 
electroactive species of the redox probe analyte, or by a non-uniform current distribution at the electrode surface due to the architecture of the electrode.

Following literature references for the modeling of impedance of thin film electrodes $[40,41]$, the equivalent circuit chosen (figure 4.b) gave the best fit results (chi-squared $\approx 10^{-3}$ ) when modeled using numerical software analysis. This impedance model is similar to the Voigt's structure [42] and consists of a resistance, $\mathrm{Re}$, and two RC circuits in series, in which the second capacitor is replaced by a diffusion-related constant phase element (CPE) ( $n=0.5 \pm \varepsilon$, where $0<\varepsilon \leq 0.1$ ). R2 represents the adsorption or diffusion resistance.

The specified values of the rate constant of electron transfer $\left(k^{0}\right)$ are calculated using equation 1 [43].

$R_{c t}=\frac{R T}{n^{2} F^{2} k^{0} c_{0} A}$

where $R_{c t}$ is the charge transfer resistance. The high values of $R_{c t}\left(136 \mathrm{k} \Omega \mathrm{cm}^{2}\right)$ and $R_{2} \quad(146$ $\mathrm{k} \Omega \mathrm{cm}^{2}$ ) indicate a diffusion limited process of the impedance response and more outer-sphere electrode reactions occur, which means that the reactant does not interact strongly with the electrode surface. The low rate constant calculated $\left(\mathrm{k}^{0}<1 \times 10^{-6} \mathrm{~cm} / \mathrm{s}\right)$ confirms the sluggish electron transfer kinetics and point to a small exchange current. The charge transfer resistance can be decreased by increasing the electrode surface area, or by the application of overpotential for the activation of charge transfer, which will increase the current and double layer capacitance [10].

In figure 5.a, the simultaneous response of Au disk $\mu$ Es to $10 \mathrm{mM}$ RuRex at a scan rate of $10 \mathrm{mVs}^{-1}$ is shown. The wave shape is sigmoidal and the measured currents are in the lower nA range. The Tomes potential of $57.1 \pm 1.9 \mathrm{mV}$ indicates a reversible system. This criteria is calculated based on the equation $2[44]$.

$\left|E_{3 / 4}-E_{1 / 4}\right|=\frac{R T}{n F} \ln 9=\frac{56.45 m V}{n}$ at $25^{\circ} \mathrm{C}$

where $E_{3} / 4$ and $E_{1} / 4$ are the potential where $i=3 i_{p} / 4$ and $i=1 i p / 4$ respectively, $n$ is the number of electrons appearing in the half-reaction for the redox couple, $T$ and $F$ have their usual meaning. The steady state peak current ( $i p)$ of $77.9 \pm 1.6 \mathrm{nA}$ is similar to the theoretical diffusion-limited plateau current of $79.3 \mathrm{nA}(\mathrm{n}=9)$, calculated with the equation 3 [45].

$i_{p}=4 n F D c_{0} r$ 
where $c_{0}$ is the bulk concentration, $r$ is the radius of the disk electrode and $D$ is the analyte diffusion coefficient with value of $D=7.9 \times 10^{-10} \mathrm{~m}^{2} \mathrm{~s}^{-1}$ for RuRex [31].

The figure 5.b shows the current response of Au disk $\mu \mathrm{Es}$ to $10 \mathrm{mM}$ RuRex at a scan rate of $50 \mathrm{mVs}^{-1}$. This reversible response confirm the use of the "on chip" gold quasi reference, which does not maintain a constant potential but varies predictably $(\approx \pm 30 \mathrm{mV})$. Advantages over systems with an external $\mathrm{Ag} / \mathrm{AgCl}$ reference electrode are ease of fabrication, reduction of fabrication costs, and a better electrochemical robustness $[46,47]$. With respect to the macro electrode behavior (figure 5.c), the measured linear-diffusion peak current is $12.67 \pm 1.39 \mu \mathrm{A}(\mathrm{n}=12)$, which is similar to the calculated current response of $13.58 \mu \mathrm{A}$ based on the Randles-Sevcik equation [10]. The current ratio between anodic and cathodic peak current is $\mathrm{ipa} / \mathrm{ipc}=12.8 / 12.6=1.02$. The potential difference between the peak potential and the half peak potential is $59.1 \pm 2.8 \mathrm{mV}$. The chronoamperometry results (fig. 5.d) demonstrate the functionality of the fabricated electrodes as an electrochemical sensor. The experimental values correlate well with the theoretical value, which gives a measurement accuracy of $96.01 \%$ (external setup) and $94.94 \%$ (internal setup).

In summary, both, micro and macro electrode have a reversible electron-transfer mechanism with RuRex, which makes them suitable for amperometric applications.

\section{Conclusions}

Using the low cost, high performance polymer poly(ethylene-2,6-naphthalate) as a substrate material for $\mathrm{Au} \mu \mathrm{Es}$, a powerful electrochemical platform is developed and characterized. Importantly, a novel room-temperature passivation process is elaborated. The metal to polymer adhesion is physically and chemically robust and withstands commonly used electrochemical biosensing techniques. From the resulting data the information on charge transfer resistance, rate constant of electron transfer and reversibility of the current response can be extracted; demonstrating the robust functionality of sensor platform for investigating innovative electrochemical biosensor applications. Using antigen- antibody binding, DNA hybridization and protein-ligand binding, possible application areas could include human healthcare, security diagnostics, veterinary care and food monitoring. Supporting data demonstrating the flexibility of the system will be required, but the wide measurement range could make it particularly attractive. 
Published in Sensors and Actuators B: Chemical, V 161 (1), 3 January 2012, pp 279-284

European FP6 project DVT-IMP (Deep Vein Thrombosis-Impedimetric Microanalysis System) for funding, Dr. Josep Calderer Cardona for his honorable help in metal deposition and David Izquierdo Garcia for the help in fabrication. 


\section{Figures}

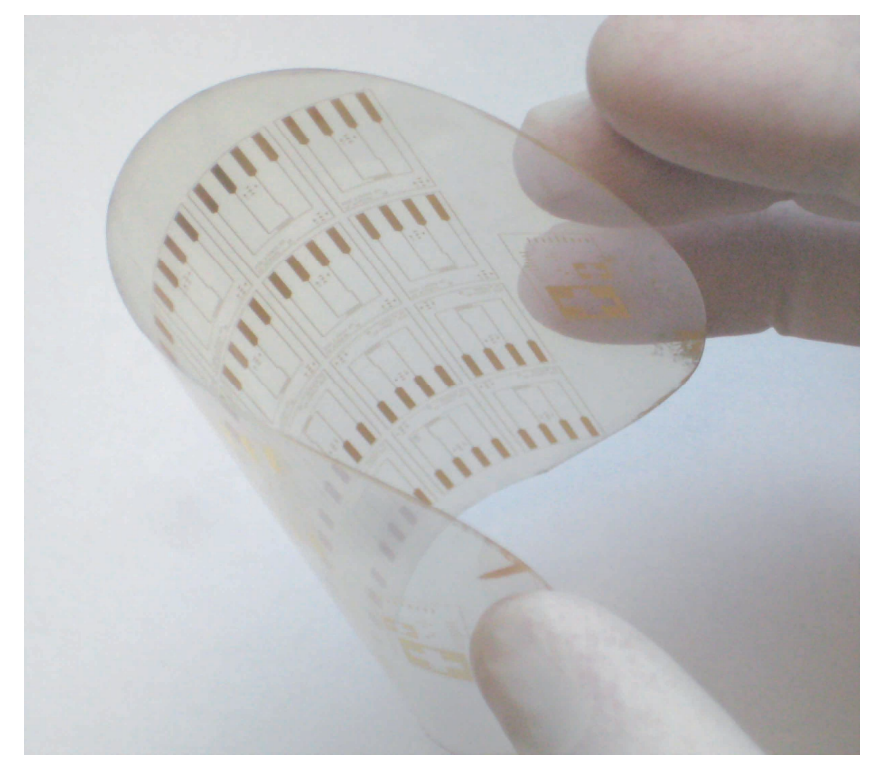

Fig. 1: Gold electrodes on PEN with a minimum feature size of $6 \pm 1 \mu \mathrm{m}$ over a maximum substrate size of $10 \mathrm{~cm}$ in diameter. 
Published in Sensors and Actuators B: Chemical, V 161 (1), 3 January 2012, pp 279-284

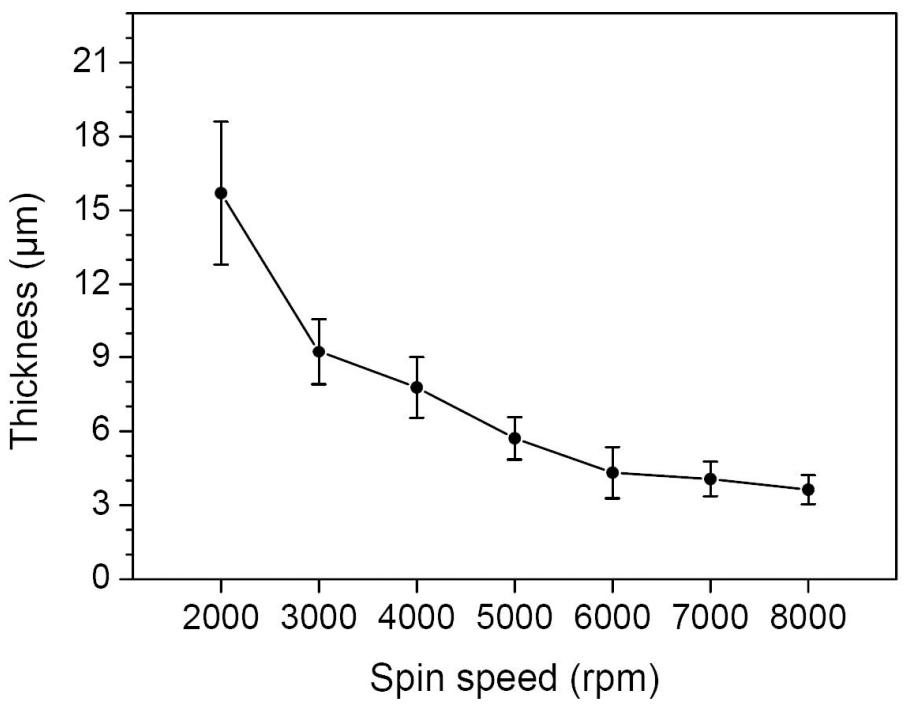

Fig. 2: Spin curve of 60-7155 UV epoxy layer on PEN. Epoxy layer thicknesses range is $15.7 \mu \mathrm{m} \pm 2.9 \mu \mathrm{m}$ to $3.6 \mu \mathrm{m} \pm 0.6 \mu \mathrm{m}$. 


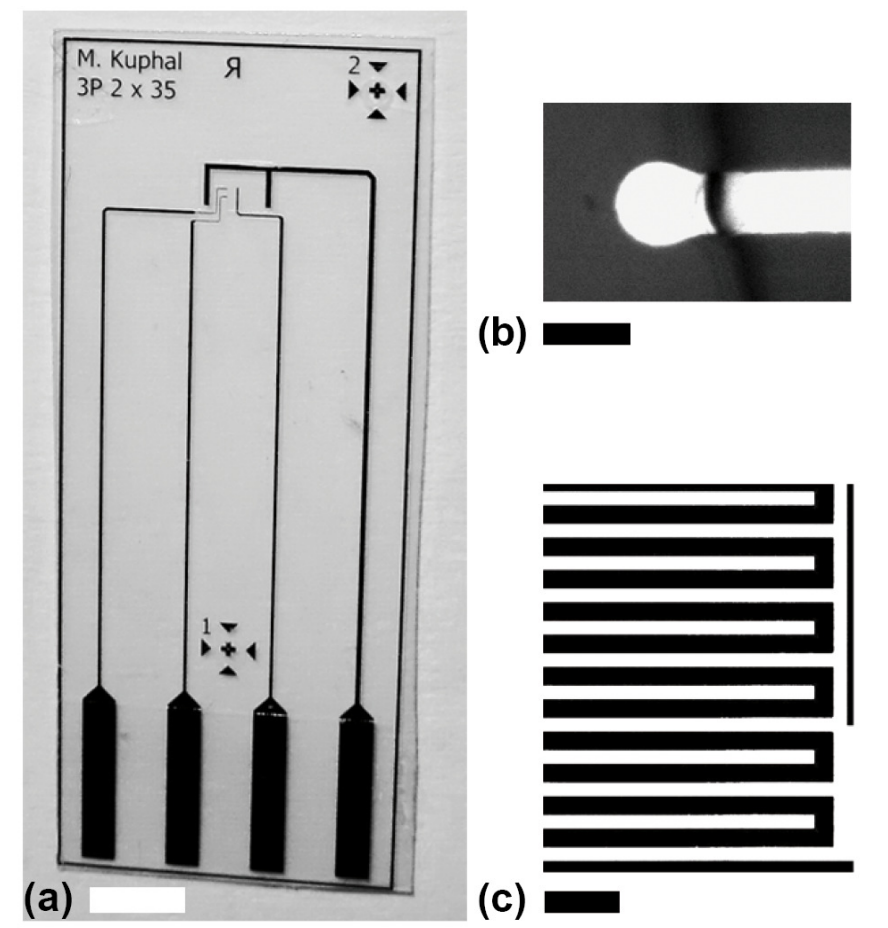

Fig. 3: (a) Image of a fabricated chip on PEN with two gold $\mu \mathrm{E}$, a gold quasi reference electrode and counter electrode (adapted from [31]) [bar $=3 \mathrm{~mm}$ ]. (b) Phase-contrast images of passivated gold $\mu \mathrm{E}$ of $30 \mu \mathrm{m}$ on PEN. The dark line vertically is the border line between the opened area (left) and the passivated area (right). [bar $=30 \mu \mathrm{m}$ ] (c) Phase-contrast images of gold IDE on PEN with width and spacing of the IDE elements of $6 \pm 1 \mu \mathrm{m}$. [bar $=30 \mu \mathrm{m}$ ] 


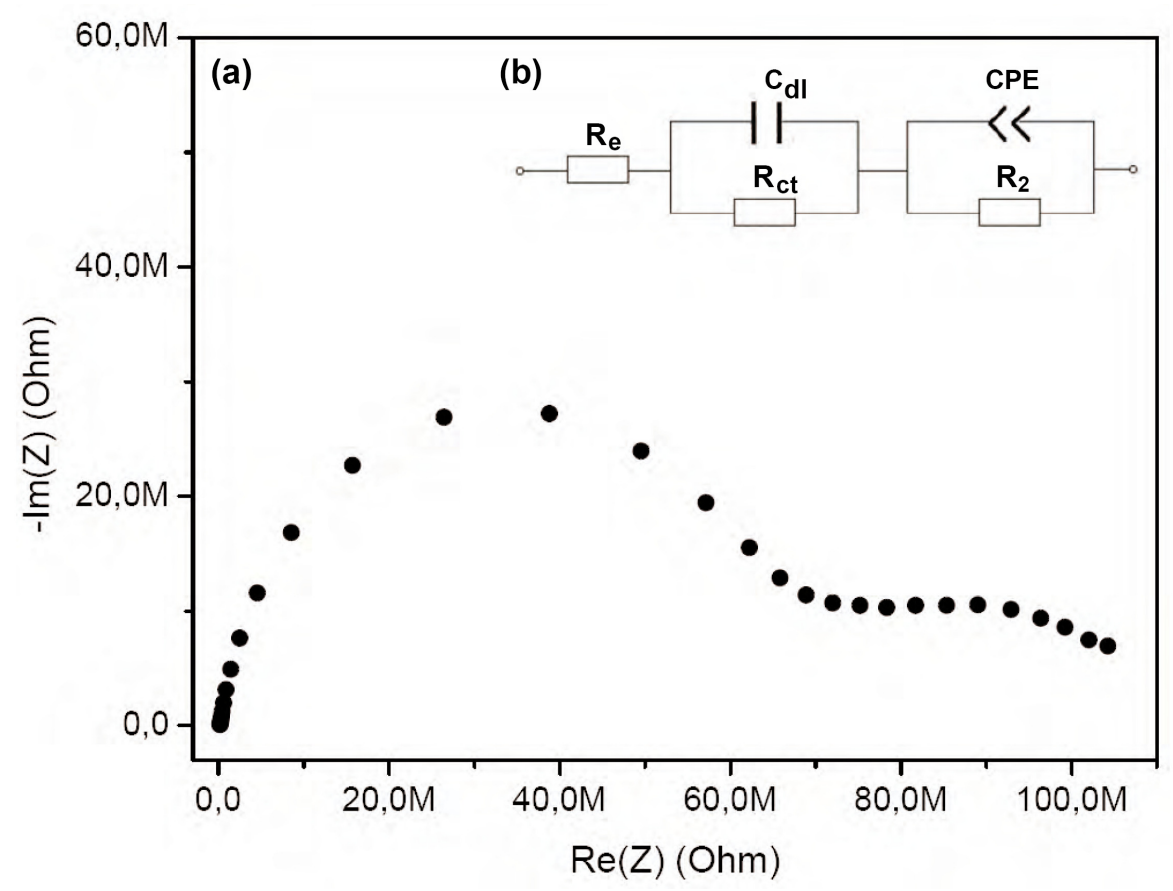

Fig. 4: (a) Nyquist plot of EIS results for IDE 0653 device in $5 \mathrm{mM}$ potassium ferrocyanide $\left(\mathrm{K}_{4} \mathrm{Fe}(\mathrm{CN})_{6}\right)$ dissolved in PBS. Applied potential of $10 \mathrm{mV}$. Frequency range $100 \mathrm{kHz}$ to $10 \mathrm{mHz}$. (b) Equivalent circuit model for impedance spectra with $\mathrm{Re}=278.9 \mathrm{Wcm}^{2}, \mathrm{Rct}=136.0 \times 10^{3} \mathrm{Wcm}^{2}, \mathrm{R}_{2}=146.0 \times 10^{3} \mathrm{Wcm}^{2}, \mathrm{Cdl}=$ $16.3 \times 10^{-12} \mathrm{~F} / \mathrm{cm}^{2}, \mathrm{CPE}=54.0 \times 10^{-6} \mathrm{~F} / \mathrm{cm}^{2}, \mathrm{n}=0.551, \chi^{2}=3.17 \times 10^{-3}, \mathrm{k}_{0}=3.92 \times 10^{-7} \mathrm{~cm} / \mathrm{s}, W \mathrm{E}_{\text {area }}=$ $2.74 \times 10^{-3} \mathrm{~cm}^{2}$ 

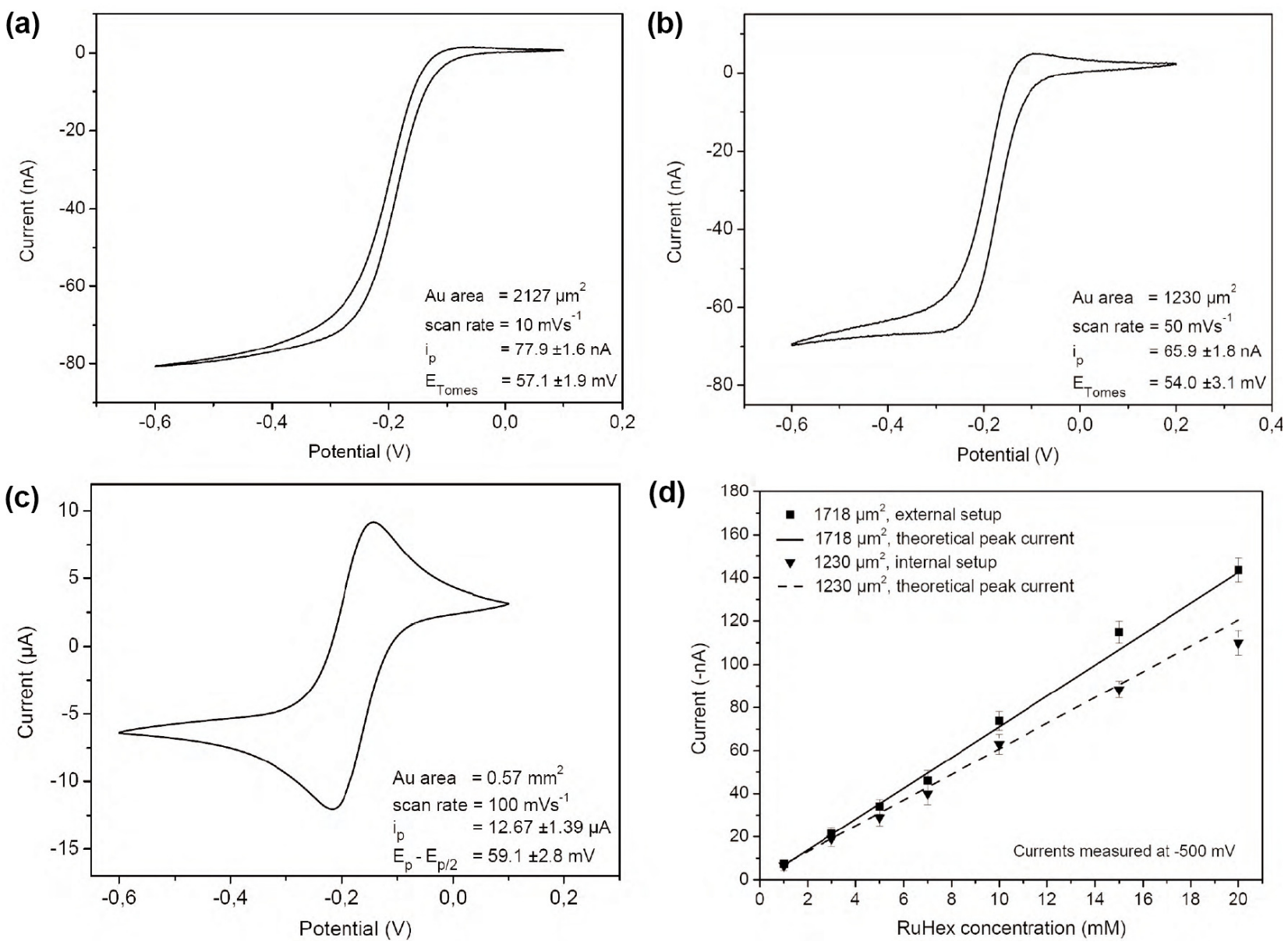

Fig. 5: Reversible cyclic voltammogram of a gold $\mu \mathrm{E}$ in $10 \mathrm{mM}$ RuHex, applied potential between $0.1 \mathrm{~V}$ and $-0.6 \mathrm{~V}$ (a) external setup, active area of $2127 \mu \mathrm{m}^{2}$ (microelectrode), scan rate of $10 \mathrm{mVs}^{-1}$, Tomes potential is $57.1 \pm 1.9 \mathrm{mV}$, ip is $77.9 \pm 1.6 \mathrm{nA}$ (theoretical ip is $79.3 \mathrm{nA}$ ), (b) internal setup, active area of $1230 \mu \mathrm{m}^{2}$, scan rate of $50 \mathrm{mVs}^{-1}$, Tome^s potential is $54.0 \pm 3.1 \mathrm{mV}$, ip is $65.9 \pm 1.8 \mathrm{nA}$ (theoretical ip is $60.3 \mathrm{nA}$ ). (c) external setup, active area of $0.576 \mathrm{~mm}^{2}$ (macro electrode), scan rate of $100 \mathrm{mVs}^{-1}$, half peak potential is $59.1 \pm 2.8 \mathrm{mV}$, ip is $12.67 \pm 1.39 \mu \mathrm{A}$ (theoretical ip is $13.58 \mu \mathrm{A}$ ). (d) Chronoamperometry response to increasing molarities of RuHex ( 1 to $20 \mathrm{mM}$ ) in $1 \mathrm{M} \mathrm{KCl}$, with an applied potential of -500 $\mathrm{mV}$, for the external and internal setup. Results compared to theoretical peak current. 
Published in Sensors and Actuators B: Chemical, V 161 (1), 3 January 2012, pp 279-284

References

[1] A. Warsinke, 2009. doi:10.1007/s00216-008-2572-0.

[2] M. C. Choi, 2008. doi:10.1016/j.progpolymsci.2007.11.004. [3] K. Nomura, 2004. doi:10.1038/nature03090.

[4] M. Peter, 2009. doi:10.1016/j.tsf.2008.11.096.

[5] O. A. Sadik, 2009. doi:10.1016/j.bios.2008.10.003. [6] D. Luo, 2009.

doi:10.1016/j.elecom.2009.03.011. [7] J. Raoof, 2010. doi:10.1007/s10008-009-0917-z.

[8] T. Ignat, 2010. doi:10.1016/j.mseb.2009.11.021. [9] T. Y. Yeo, 2011.

doi:10.1016/j.bios.2011.07.010.

[10] A. J. Bard, Electrochemical Methods, Fundamentals and Applications, Wiley, ISBN-13: 978-0-47104372-0, 2001. [11] M. E. Sandison, 2002. doi:10.1021/ac025649w.

[12] D. T. Price, 2009. doi:10.1016/j.bios.2008.10.026.

[13] D. Hoffmann, 2010. doi:10.1088/0964-1726/19/11/115016. [14] K. Long, 2006. doi:10.1109/LED.2005.863147.

[15] W. A. MacDonald, 2004. doi:10.1039/b310846p. [16] T. Stieglitz, 2000. doi:10.1023/A:1009955222114.

[17] A. E. Tonelli, 2002. doi:10.1016/81089-3156(00)00028-3. [18] S. Yasufuku, 2003. doi:10.1109/ME!.2003.1266349.

[19] T. Mäkelä, 2005. doi:10.1016/j.synthmet.2005.07.140.

[20] W. S. Wong, 2006. doi:10.1016/j.jnoncrysol.2005.12.055. [21] M. Fonrodona, 2005. doi:10.1016/j.solmat.2004.12.006. [22] K. Sidler, 2008. doi:10.1016/j.mee.2007.12.069.

[23] S. K. Bhagat, 2008. doi:10.1016/j.tsf.2007.12.146. [24] T. N. Ng, 2007. doi:10.1063/1.2767981.

[25] M. Peter, 2009. doi:10.1021/la8037457.

[26] E. Valera, 2009. doi:10.1016/j.mee.2009.07.001.

[27] N. Hameed, 2007. doi:10.3144/expresspolymlett.2007.49. [28] S. Kisin, 2007. doi:10.1016/j.tsf.2007.02.018.

[29] A. V. Mamishev, 2004. doi:10.1109/JPROC.2004.826603. 
[30] R. G. Compton, 2005. doi:10.1016/j.jelechem.2005.07.022. [31] A. P. Bhavik, 2008. doi:10.1007/s00216-007-1803-0.

[32] J. Israelachvili, Intermolecular and Surface forces, Elsevier, ISBN: 0-12-375181-0, 1991. [33] S. J. Chua, 2008. doi:10.4028/www.scientific.net/AMR.31.30.

[34] C. P. Wong, 2003. doi:10.1109/TEPM.2003.822996. [35] D. Simeone, 2009. doi:10.1016/j.tsf.2009.02.088.

[36] W. S. Lau, 2007. doi:10.1016/j.microrel.2007.05.003. [37] P. E. Ganrou, 2002. doi:10.1109/6040.784503.

[38] H. Sharifi, 2009. doi:10.1109/TADVP.2008.2006760. [39] A. Olziersky, 2010. doi:10.1063/1.3477192.

[40] N. Ariel, 2005. doi:10.1063/1.1989431. [41] K. Y. Tse, 2005. doi:10.1021/jp046433w.

[42] D. Vladikova, 2002. doi:10.1016/80013-4686(02)00187-1. [43] I. Dumitrescu, 2009. doi:10.1016/j.elecom.2009.08.057. [44] K. B. Oldham, 1989. doi:10.1016/0022-0728(89)85029-6. [45] S. Bruckenstein, 2002. doi:10.1016/80022-0728(02)01251-2. [46] S. B. Prakash, 2008. doi:10.1016/j.snb.2007.09.029.

[47] X. Hu, 2010. doi:10.1016/j.jelechem.2009.10.026. 